\title{
Deletion 14q (q22q23) associated with anophthalmia, absent pituitary, and other abnormalities
}

\author{
C P Bennett, David R Betts, Mary J Seller
}

\begin{abstract}
A fetus is described with anophthalmia, absent pituitary, hypoplastic adrenal glands and kidneys, absent left horn of the uterus, underdeveloped genitalia, and clinodactyly, with a deletion of 14(q22q23). A review of published reports found no similar deletion cases.
\end{abstract}

Although deletions of small chromosomal segments are relatively common, there are only a few instances reported of deletions involving the long arm of chromosome 14. Furthermore, most of these are terminal; there appear to be only four published cases of interstitial deletions. ${ }^{1-3} \mathrm{We}$ describe a fetus with an interstitial deletion of $14 \mathrm{q}$ and an unusual combination of clinical features.

\section{Case report}

This was the fourth pregnancy of unrelated Caucasians, the mother and father being 31 and 29 years old, respectively. The first pregnancy was ectopic, the second ended as a spontaneous abortion at 12 weeks, and the third resulted in a normal appearing stillbirth at 22 weeks owing to placenta praevia. In the fourth pregnancy, ultrasound examination at 21 weeks showed an unusually shaped fetal head, small orbits with the absence of eye lenses, loose skin at the back of the neck, and possible polydactyly. In view of these findings, the couple opted for termination of the pregnancy.

The female fetus was markedly dysmorphic (figure). There was bilateral anophthalmia, confirmed by histology, and short, widely spaced palpebral

\footnotetext{
Department of Medical and Molecular Genetics, United Medical and Dental Schools of Guy's and St Thomas's Hospitals, 8th Floor, Guy's Tower, London SE1 9RT. C P Bennett, D R Betts*, M J Seller

*Present address: ICRF Department of Medical Oncology, St Bartholomew's Hospital, London.

Correspondence to Dr Bennett.
}

Received for publication 1 October 1990 Accepted for publication 5 October 1990.

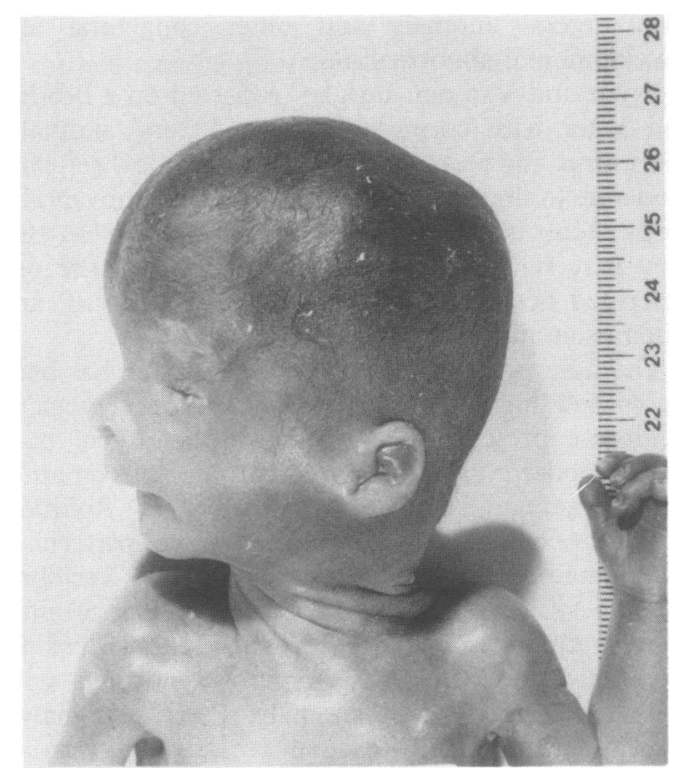

Twenty-one week fetus showing anophthalmia, brachycephaly, abrupt occiput, high forehead, anteverted, flared nostrils, upper lip markedly overriding the lower lip, receding lower jaw, low set, fleshy ears, and excess skin on neck.

fissures. The nose had a high bridge and anteverted and flared nares. The upper lip markedly overrode the lower and the lower jaw was receding. The ears were fleshy, angular, and low set. There was brachycephaly with an abrupt vertical occiput, and the forehead was high and prominent. There was excess skin around the posterior neck. On both hands the fifth fingers were incurved with short middle phalanges and the thumbs were flexed. The external genitalia were underdeveloped.

Internally, the left uterine horn was absent, the kidneys were somewhat small, and the adrenal glands markedly hypoplastic. Histologically, the kidneys appeared hypoplastic and the adrenal glands had a reduced fetal cortical layer, similar to that seen in anencephaly. There were abnormalities of the cranium and brain. The cranial floor was short in the anterior and posterior direction and the pituitary fossa 
was absent. The optic nerves, chiasma and tracts, the geniculate bodies, pituitary stalk and gland were absent; the cerebellum was small.

Tissue from the fetus was cultured and showed a small deletion of the long arm of chromosome 14, karyotype 46,XX,del(14)(q22q23). Both parents had normal chromosomes.

\section{Discussion}

The four other cases of interstitial deletions of $14 q^{1-3}$ had breakpoints more distal than those of the present case (table). There is no consistent clinical picture in these four patients, although all were dysmorphic, with overdeveloped eyebrows, a small, bulbous nose, micrognathia, developmental delay, and mental retardation. One (case 1$)^{1}$ had a horseshoe kidney and an atrial septal defect (ASD), another (case 2) ${ }^{1}$ was said to have Holt-Oram syndrome with reduction deformities of the forearms and thumbs and an ASD, and yet another (case 3$)^{2}$ also had a cleft palate.

Two chromosome aberrations involving regions of $14 \mathrm{q}$ more proximal than our case have been reported. One $^{4}$ was a complex translocation involving chromosomes 3,11 , and 14 which included an interstitial deletion of $14 \mathrm{q}$ at q13q22. The child had bilateral cleft lip, complete cleft palate, and congenital glaucoma. The other ${ }^{5}$ resembled our case in one respect; the child had bilateral anophthalmia, but also unilateral macrostomia, preauricular skin tags, and cervical hemivertebrae, and was associated with a translocation between chromosomes 4 and 14, with the breakpoint on $14 \mathrm{q}$ at q11.

The combination of defects found in our fetus is

Details of the interstitial deletion breakpoints of $14 q$.

\begin{tabular}{lcl}
\hline Case & Reference & \multicolumn{1}{c}{ Karyotype } \\
\hline 1 & 1 & $46, X Y, \operatorname{del}(14)(q 23 q 32)$ \\
2 & 1 & $46, X Y$,del(14)(q23q24.2) \\
3 & 2 & $46, X Y$,del(14)(q24.3q32.1) \\
4 & 3 & $46, X Y$,del(14)(q24.3q32.1) \\
5 & & $46, X X$,del(14)(q22q23) \\
\hline
\end{tabular}

unusual. A search of the London Dysmorphology Database and McKusick's Mendelian inheritance in man has shown no similar case.

Primary anophthalmia (no eye tissue in the orbit) with no other associated features is an autosomal recessive condition. Anophthalmia in conjunction with other defects can be found in Fraser syndrome, Goldenhaar syndrome, and anophthalmia (type Waardenberg), but in these there is no pituitary abnormality. Absent pituitary gland can be seen in holoprosencephaly, but this is not associated with anophthalmia. There is, however, a report of five mentally retarded adults ${ }^{6}$ with microphthalmia or anophthalmia and hypogonadism with low gonadotrophin levels, which suggests a hypothalamic or pituitary deficit. Their chromosomes were said to be normal.

This combination of abnormalities in our case appears to be unique, and the additional finding of a specific minute chromosomal deletion suggests that this region of the genome on 14q, q22q23, could be important for normal eye, pituitary, craniofacial, and uterine development.

We thank Miss Raju, Consultant Obstetrician, for referring this patient, Dr N Fagg for the histology, and the Spastics Society for financial support.

1 Turleau C, de Grouchy J, Chavin-Colin F, et al. Two patients with interstitial del(14q), one with features of Holt-Oram syndrome. Exclusion mapping of PI (alpha-1-antitrypsin). Ann Genet (Paris) 1984;27:237-40.

2 Kawamura G, Suzuki M, Segawa T, et al. A case of partial monosomy 14q. J Pediatr Pract $(\Im p n)$ 1985;48:32-4.

3 Yamamoto Y, Sawa R, Okamoto N, et al. Deletion 14q(q24.3 to q32.1) syndrome: significance of peculiar facial appearance in its diagnosis, and deletion mapping of $\mathrm{Pi}$ ( $\alpha_{1}$-antitrypsin). Hum Genet 1986;74:190-2.

4 Buchanan PD, Rao KW, Doerr CL, et al. A complex translocation involving chromosomes 3,11 and 14 with an interstitial deletion, del(14)(q13q22) in a child with congenital glaucoma and cleft lip and palate. In: Birth defects. Vol 14, No 6c. New York: Alan R Liss, 1978:317-22.

5 Serville F, Broustet A, Peyeresblangues J, et al. Anophthalmie bilaterale, anomalies de la face et translocation $t(4 ; 14)$. F Genet Hum 1974;22:341-51.

6 Keppen LD, Brodsky MC, Michael JM, et al. Hypogonadotropic hypogonadism in mentally retarded adults with microphthalmia and clinical anophthalmia. Am $\mathcal{J}$ Med Genet 1990;36:285-7. 